Pacific Journal of Mathematics

SOME NUMBER THEORETIC RESULTS 


\title{
SOME NUMBER THEORETIC RESULTS
}

(In memory of our good friend Leo Moser)

\author{
P. ERdös and E. G. Straus
}

The paper first establishes the order of magnitude of maximal sets, $S$, of residues $(\bmod p)$ so that the sums of different numbers of elements are distinct.

In the second part irrationalities of Lambert Series of the form $\sum f(n) / a_{1} \cdots a_{n}$ are obtained where $f(n)=d(n), \sigma(n)$ or $\varphi(n)$ and the $a_{i}$ are integers, $a_{i} \geqq 2$, which satisfy suitable growth conditions.

This note consists of two rather separate topics. In $\S 1$ we generalize a topic from combinatorial number theory to get an order of magnitude for the number of elements in a maximal set of residues $(\bmod p)$ such that sums of different numbers of elements from this set are distinct. We show that the correct order is $c p^{1 / 3}$ although we are unable to establish the correct value for the constant $c$.

Section 2 consists of irrationality results on series of the form $\Sigma f(n) / a_{1} a_{2} \cdots a_{n}$ where $f(n)$ is one of the number theoretic functions $d(n), \sigma(n)$ or $\varphi(n)$ and $a_{n}$ are integers $\geqq 2$. For $f(n)=d(n)$ it suffices that the $\alpha_{n}$ are monotonic while for $\sigma(n)$ and $\varphi(n)$ we needed additional conditions on their rates of growth.

1. Maximal sets in a cyclic group of prime order for which subsets of different orders have different sums. In an earlier paper [4] one of us has given a partial answer to the question:

What is the maximal number $n=f(x)$ of integers $a_{1}, \cdots, a_{n}$ so that $0<a_{1}<a_{2}<\cdots<a_{n} \leqq x$ and so that

$$
\begin{aligned}
& a_{i_{1}}+\cdots+a_{i_{s}}=a_{j_{1}}+\cdots+a_{j_{t}} \text { for some } 1 \leqq i_{1}<\cdots<i_{s} \leqq n \\
& 1 \leqq j_{1}<\cdots<j_{t} \leqq n
\end{aligned}
$$

implies $s=t$ ? it is conjectured that the maximal set is obtained (loosely speaking) by taking the top $2 \sqrt{x}$ integers of the interval $(1, x)$. We were indeed able to prove that $f(x)<c \sqrt{x}$ for suitable $c$ (for example $4 / \sqrt{3}$ ) by using the fact that a set of $n$ positive integers has a minimal set of distinct sums of $t$-tuples $(1 \leqq t \leqq n)$ if it is in arithmetic progression.

It is natural to pose the analogous question for elements of cyclic groups of prime order, as was done at the Number Theory Symposium in Stony Brook [5]. Here again we may conjecture that a maximal set of residues $(\bmod p)$ is attained by taking a set of consecutive residues, this time not at the upper end but near $p^{2 / 3}$. 
Conjecture 1.1. Let $f(p)$ be the maximal cardinality of a set. of residues mod $p$ so that sums of different numbers of residues in this set are different, then $f(p)=(4 p)^{1 / 3}+o\left(p^{1 / 3}\right)$ where the maximum is attained, for example, by taking consecutive residues in an interval of length $(4 p)^{1 / 3}+o\left(p^{1 / 3}\right)$ containing the residue $\left[(p / 2)^{1 / 3}\right]$.

It is easy to see that we can indeed get a set of about $(4 p)^{1 / 3}$ residues by taking the residues in the interval $\left(\left[(p / 2)^{2 / 3}-(4 p)^{1 / 3}\right]\right.$, $\left.\left[(p / 2)^{2 / 3}\right]\right)$. Here sums of distinct numbers of elements are distinct integers, and since all sums are $<p$ it follows that they are distinct residues.

The observation which let to the upper bound in [4] is much less obvious $(\bmod p)$ :

Conjecture 1.2. A set $A=\left\{a_{1}, a_{2}, \cdots, a_{k}\right\}$ of residues $(\bmod p)$ has a minimal number of distinct sums of subsets of $t$ elements if $A$ is in arithmetic progression.

Conjecture 1.2 would give us a simple upper bound for $f(p)$ :

COROLlaRY 1.3. If Conjecture 1.2 holds then

$$
f(p)<(6 p)^{1 / 3}+o\left(p^{1 / 3}\right) .
$$

Proof. The sums of $t$ elements from the set of residues

$$
\{1,2, \cdots, k-1, k\}
$$

fill the interval $\left(\left(\begin{array}{c}t+1 \\ 2\end{array}\right), t k-\left(\begin{array}{l}t \\ 2\end{array}\right)\right)$ that is to say there are $t k-t^{2}+O(t)$ such sums. Since for different $t$ we get different sums we must have

$$
\begin{aligned}
& p \geqq \sum_{t=1}^{k}\left(t k-t^{2}+O(t)\right)=\frac{k^{3}}{6}+O\left(k^{2}\right) \\
& \text { and hence } k<(6 p)^{1 / 3}+o\left(p^{1 / 3}\right) .
\end{aligned}
$$

Using methods employed by Erdös and Heilbronn [2] we can show that $f(p)=O\left(p^{1 / 3}\right)$. We use the following lemma from [2].

Lemma 1.4. Let $1<m \leqq l<p / 2$ and let $B=\left\{b_{1}, \cdots, b_{l}\right\}, A=$ $\left\{a_{1}, \cdots, a_{m}\right\}$ be sets of residues $(\bmod p)$. Then there exists an $a_{i} \in A$ such that the number of solutions of $a_{i}=b_{j}-b_{k} ; b_{j}, b_{k} \in B$ is less than $l-m / 6$.

We now can get a lower bound for the number of distinct sums of $t$ elements from a set of residues.

Lemma 1.5. Let $A=\left\{a_{1}, \cdots, a_{k}\right\}$ be a set of residues $(\bmod p)$ 
and let $A_{t}=\left\{a_{i_{1}}+\cdots+a_{i_{t}} \mid 1 \leqq i_{1}<\cdots<i_{t} \leqq k\right\}$ then for $1 \leqq t \leqq k / 4$ we have

$$
\left|A_{t}\right| \geqq l+\frac{(t-1) m}{6}-\frac{t(t-1)}{6}
$$

where

$$
l=\left[\frac{k+1}{2}\right], m=\left[\frac{k}{2}\right]
$$

Proof. We divide the set $A$ into two disjoint sets

$$
A=\left\{a_{1}, a_{2}, \cdots, a_{l}\right\}, B=\left\{b_{1}, b_{2}, \cdots, b_{m}\right\}
$$

and prove the inequality (1.6) for the subset of $A_{t}$ consisting of the sums

$$
A_{t}^{*}=\left\{a_{i}+b_{2-\varepsilon_{1}}+b_{4-\varepsilon_{2}}+\cdots+b_{2 t-2-\varepsilon_{t-1}} \mid \varepsilon_{j}=0 \text { or } 1\right\},
$$

where the $b_{i}$ are a suitable ordering of the elements of $B$.

The inequality holds for $t=1$ since

$$
A_{t}^{*}=\left\{a_{i}\right\}=A \text { and }|A|=l .
$$

Now assume that (1.6) holds for $A_{t}{ }^{*}$ with $t \leqq(m / 2)-1$. Then the set $A_{t}{ }^{*}+b_{2 t} \subset A^{*}{ }_{t+1}$ and according to Lemma 1.3 there exists Ia $b_{j} \in\left\{b_{2 t+1}, b_{2 t+1}, \cdots, b_{m}\right\}$, say $b_{j}=b_{2 t+1}$ so that the equation

$$
b_{2 t+1}-b_{2 t}=a_{i}^{*}-a_{j}^{*}, a_{i}^{*}, a_{j}^{*} \in A_{t}^{*}
$$

has no more than $\left|A_{t}^{*}\right|-\frac{1}{8}(m-2 t)$ solutions. Hence the set

$$
\left(\left(b_{2 t+1}-b_{2 t}\right)+\left(A_{t}^{*}+b_{2 t}\right)\right) \cap\left(A_{t}^{*}+b_{2 t}\right)
$$

contains no more than $A_{t}^{*}-\frac{1}{8}(m-2 t)$ elements and

$$
\begin{aligned}
\left|A_{t+1}^{*}\right| & =\left|\left(A_{t}^{*}+b_{t+1}\right) \cup\left(A_{t}^{*}+b_{t}\right)\right| \\
& \geqq\left|A_{t}^{*}\right|+\frac{1}{6}(m-2 t) \\
& \geqq l+\frac{(t-1) m}{6}-\frac{t(t-1)}{6}+\frac{1}{6} m-\frac{t}{3} \\
& =l+\frac{t m}{6}-\frac{(t+1) t}{6} .
\end{aligned}
$$

This completes the proof.

THEOREM 1.7. The maximal number $f(p)$ of a set $A$ of residues $(\bmod p)$ so that sums of different numbers of distinct elements of $A$ are distinct satisfies

$$
(4 p)^{1 / 3}+o\left(p^{1 / 3}\right)<f(p)<(288 p)^{1 / 3}+o\left(p^{1 / 3}\right) .
$$


Proof. According to Lemma 1.5 there are at least

$$
k / 2+k(t-1) / 12-t^{2} / 6+O(t)
$$

distinct sums of $t$ elements (and hence, by symmetry, sums of $k-t$ elements) for $t<[k / 4]$ out of a set $A$ with $k$ elements. Thus if $A$ has the desired property we must have

$$
\begin{aligned}
p & \geqq 2 \sum_{t=1}^{k / 4}\left(k / 2+k(t-1) / 12-t^{2} / 6\right)+O\left(k^{2}\right) \\
& =2 k^{3}\left(\frac{1}{384}-\frac{1}{3} \frac{1}{384}\right)+O\left(k^{2}\right)=k^{3} / 288+O\left(k^{2}\right) .
\end{aligned}
$$

Thus

$$
f(p)<(288 p)^{1 / 3}+o\left(p^{1 / 3}\right) .
$$

The lower bound for $f(p)$ was established above.

2. On some irrational series. One of us [1] proved that the series $\sum_{n=1}^{\infty} d(n) t^{-n}$ is irrational for every integer $t,|t|>1$. In this section we generalize this result to series of the form

$$
\xi=\sum_{n=1}^{\infty} \frac{d(n)}{a_{1} a_{2} \cdots a_{n}}
$$

where the $a_{n}$ are positive integers with $2 \leqq a_{1} \leqq a_{2} \leqq \cdots$. It is clear that we need some restriction, such as monotonicity, on the $a_{n}$ since the choice $a_{n}=d(n)+1$ would lead to $\xi=1$.

We divide the proof into two cases depending on the rate of increase of $a_{n}$. The first case is very similar to [1].

LEMMA 2.2. The series (2.1) is irrational if there exists $a \delta>0$ so that the inequality $a_{n}<(\log n)^{1-\delta}$ holds for infinitely many values of $n$.

Proof. Let $n$ be a large integer so that $a_{n}<(\log n)^{1-\hat{o}}$. Then by the monotonicity of $a_{i}$ there exists an interval $I$ of length $n / \log n$ in $(1, n)$ so that for all integers $i \in I$ we have $a_{i}=t$ where $t$ is a fixed integer, $t \leqq(\log n)^{1-\delta}$.

Now put $k=\left[(\log n)^{\delta / 10}\right]$ and let $p_{1}, p_{2}, \cdots$ be the consecutive primes greater than $(\log n)^{2}$. Let

$$
A=\left(\prod_{1 \leqq i \leqq k(k+1) / 2} p_{i}\right)^{t}
$$

then

$$
\begin{gathered}
A<\left(2(\log n)^{2}\right)^{t k(k+1) / 2}<e^{(\log n)^{1-\delta}(\log n)^{\hat{o} / 4}} \\
<e^{(\log n)^{1-\delta / 2}} .
\end{gathered}
$$


By the Chinese remainder theorem the congruences

$$
\begin{aligned}
x & \equiv p_{1}{ }^{t-1}\left(\bmod p_{1}{ }^{t}\right) \\
x+1 & \equiv\left(p_{2} p_{3}\right)^{t-1}\left(\bmod \left(p_{2} p_{3}\right)^{t}\right) \\
& \vdots \\
x+k-1 & \equiv\left(p_{u} p_{u+1} \cdots p_{u+k-1}\right)^{t-1}\left(\bmod \left(p_{u} p_{u+1} \cdots p_{u+k-1}\right)^{t}\right)
\end{aligned}
$$

where $u=1+k(k-1) / 2$, have solutions determined $(\bmod A)$. The interval $I$ contains at least $[n /(A \log n)]$ solutions of (2.4).

Now assume that $\xi=a / b$ and choose $x \in I$ to be a solution of (2.4) so that $(x, x+k) \subset I$. Then

$$
\begin{aligned}
b a_{1} \cdots a_{x-1} \hat{\xi} & =\text { integer }+b \sum_{l=0}^{k-1} \frac{d(x+l)}{t^{l+1}} \\
& +b \sum_{s=0}^{\infty} \frac{d(x+k+s)}{t^{k} a_{x+k} \cdots a_{x+k+s}} .
\end{aligned}
$$

But (2.4) implies that $d(x+l) \equiv 0\left(\bmod t^{l+1}\right)$ for $l=0,1, \cdots, k-1$. Thus (2.5) implies that

$$
b a_{1} \cdots a_{x-1} \xi=\text { integer }+\frac{b}{t^{k}} \sum_{s=0}^{\infty} \frac{d(x+k+s)}{a_{x+k} \cdots a_{x+k+s}} .
$$

We now wish to show that for suitable choice of $x$ the sum on the right side of (2.6) is less than 1 and hence $b \xi$ cannot be an integer. We first consider the sum

$$
\begin{aligned}
& \frac{b}{t^{k}} \sum_{s>10 \log n} \frac{d(x+k+s)}{a_{x+k} \cdots a_{x+k+s}} \\
< & \frac{b}{t^{k}} \sum_{s>1010 g n} \frac{x+k+s}{t^{s+1}}<b(x+k) \sum_{s>10 \log n} \frac{s}{t^{s}} \\
< & \frac{2 b n}{n^{2}}<\frac{1}{2} \text { for large } n .
\end{aligned}
$$

Next we wish to show that it is possible to choose $x$ so that

$$
d(x+k+s)<2^{k / 4} \text { for } 0 \leqq s<10 \log n .
$$

We first observe that

$$
(x+k+s, A)=1 \text { for all } 0 \leqq s<10 \log n
$$

since otherwise

$$
x+k+s \equiv 0\left(\bmod p_{j}\right) \text { for some } 1 \leqq j \leqq k(k+1) / 2
$$

and

$$
x+i \equiv 0\left(\bmod p_{j}\right) \text { for some } 0 \leqq i<k \text {. }
$$


But

$$
0<k+s-i<11 \log n<(\log n)^{2}<p_{j}
$$

so that (2.10) and (2.11) are incompatible.

Let $x=x_{0}, x_{0}+A, \cdots, x_{0}+z A$ be the solutions of (2.4) for which $(x, x+k) \subset I$. From (2.9) we get

$$
\begin{aligned}
\sum_{y=0}^{z} d\left(x_{0}+k+s+y A\right) & <2 \sum_{l=1}^{\sqrt{n}}\left(\frac{n}{A l}+1\right) \\
& <c \frac{n \log n}{A} .
\end{aligned}
$$

Thus the number of $y$ 's for which $d\left(x_{0}+k+s+y A\right)>2^{k / 4}$ is less than $c n \log n /\left(A .2^{k / 4}\right)$, and the number of $y$ 's so that for some $0 \leqq s<10 \log n$ we have $d\left(x_{0}+k+s+y A\right)>2^{k / 4}$ is less than

$$
10 c n \log ^{2} n /\left(A .2^{k / 4}\right)<1 / 2 n /(A \log n)<z \text {. }
$$

It is therefore possible to choose $x=x_{0}+y A \in I$ so that (2.8) holds. For such a choice we get

$$
\begin{aligned}
\frac{b^{10}}{t^{k}} \sum_{s=0}^{\log n} \frac{d(x+k+s)}{a_{x+k} \cdots a_{x+k+s}} & <\frac{b}{t^{k}} 2^{k / 4} \sum_{s=0}^{\infty} \frac{1}{t^{s}} \\
& <b .2^{-3 k / 4}<\frac{1}{2} .
\end{aligned}
$$

Combining (2.7) and (2.13) we see that $\xi$ is irrational.

LEMMA 2.14. If there exists a positive constant $c$ so that $\left|a_{n}\right|>$ $c(\log n)^{3 / 4}$ for all $n$ then the series (2.1) is irrational.

Note that in this lemma we need not assume the monotonicity of $a_{n}$ (or even that they are positive, however for simplicity we give the proof for positive $a_{n}$ only).

Proof. We use two results. The Dirichlet divisor theorem

$$
\sum_{n=1}^{N} d(n) \sim N \log N
$$

and the average order of $d(n)$, [3]

$$
d(n)<(\log n)^{\log 2+\varepsilon} \text { for almost all } n .
$$

From (2.15) we get the following.

LEMMA 2.17. Given constants $b, c>0$, then for almost all integers $x$ 


$$
d(x+y)<b^{-1}(2 c)^{-y}(\log x)^{3 y / 4} ; y=3,4, \cdots
$$

Proof. If we choose $x$ large enough so that $\log x>(2 b c e)^{4 / 3}$ then the right side of (2.18) is greater than $e^{y}$ which exceeds $x+y$, and hence $d(x+y)$, whenever $y>2 \log x$. Thus, if (2.18) fails to hold for sufficiently large $x$ then it must fail to hold for some $y$ with $3 \leqq y \leqq 2 \log x$.

Now if there are $c_{1} N$ integers $x$ below $N$ so that (2.18) fails to hold then we have more than $c_{2} N$ integers $x$ with $\sqrt{N} \leqq x \leqq N-2 \log N$ and

$$
\begin{gathered}
d(x+y)>b^{-1}(2 c)^{-y}(\log x)^{3 y / 4} \geqq b^{-1}(2 c)^{-y}\left(\frac{1}{2} \log N\right)^{3 y / 4} \\
\geqq b^{-1}(4 c)^{-3}(\log N)^{9 / 4}=c_{3}(\log N)^{9 / 4} .
\end{gathered}
$$

Thus

$$
\begin{aligned}
\sum_{n=1}^{N} d(n) & \geqq c_{2} N \cdot \frac{1}{2 \log N} c_{3}(\log N)^{9 / 4} \\
& =c_{4} N(\log N)^{5 / 4}
\end{aligned}
$$

which contradicts (2.15) for large $N$.

Combining Lemma 2.17 with (2.16) we find that there exists an infinite set $S$ of integers $x$ so that

$$
d(x+1)<\frac{b^{-1} c}{2}(\log x)^{3 / 4}, d(x+2)<\frac{b^{-1} c^{2}}{4}(\log x)^{3 / 4}
$$

and (2.18) both hold.

Now assume that $\xi=a / b$ is a rational value of (2.1) and choose $n \in S$. Then

$$
a_{1} \cdots a_{n} b \xi=\text { integer }+b \sum_{y=1}^{\infty} \frac{d(n+y)}{a_{n+1} \cdots a_{n+y}}
$$

where

$$
0<\sum_{y=1}^{\infty} \frac{d(n+y)}{a_{n+1} \cdots a_{n+y}}<\sum_{y=1}^{\infty} \frac{(2 c)^{-y}(\log n)^{3 y / 4}}{\left(c(\log n)^{3 / 4}\right)^{y}}=1,
$$

in contradiction to the fact that the left side of (2.22) is an integer.

Summing up we have

THEOREM 2.23. The series (2.1) is irrational whenever

$$
2 \leqq a_{1} \leqq a_{2} \leqq \cdots \leqq a_{n} \leqq \cdots .
$$

With considerable additional effort one can weaken the monotonicity condition on the $a_{n}$ to $a_{m} / a_{n} \geqq c>0$ for all $m>n$.

We have not been able to prove the following 
Conjecture 2.24. The series (2.1) is irrational whenever $a_{n} \rightarrow \infty$. If we consider series of the form

$$
\sum_{n=1}^{\infty} \frac{\varphi(n)}{a_{1} \cdots a_{n}} \text { or } \quad \sum_{n=1}^{\infty} \frac{\sigma(n)}{a_{1} \cdots a_{n}}
$$

then we cannot make conjectures analogous to 2.24 since the choice $a_{n}=\varphi(n)+1$ or $\sigma(n)+1$ would make these series converge to 1 . It is reasonable to conjecture that the series (2.25) must be irrational if the $a_{n}$ increase monotonically, however we can prove this only under more restrictive conditions.

THEOREM 2.26. If $\left\{a_{n}\right\}$ is a monotonic sequence of integers with $a_{n} \geqq n^{11 / 12}$ for all large $n$ then the series in (2.25) are irrational.

For the proof we need the following simple lemmas.

LEMMA 2.27. Let $\left\{a_{n}\right\}$ be a sequence of positive integers with $a_{n} \geqq 2$ and $\left\{b_{n}\right\}$ a sequence of positive integers so that $b_{n+1}=o\left(a_{n} a_{n+1}\right)$. If

$$
\xi=\sum_{n=1}^{\infty} \frac{b_{n}}{a_{1} \cdots a_{n}}
$$

is rational then $a_{n}=O\left(b_{n}\right)$.

Proof. Assume $\xi=a / b$ and choose $N$ so that for all $n>N$ we have $b b_{n}<a_{n-1} a_{n} / 4$. If there existed an $n>N$ so that $a_{n}>2 b b_{n}$ then we would have

$$
b a_{1} \cdots a_{n-1} \xi=a a_{1} \cdots a_{n-1}=\text { integer }+\sum_{k=0}^{\infty} \frac{b b_{n+k}}{a_{n} \cdots a_{n+k}}
$$

but

$$
\begin{aligned}
0<\sum_{k=0}^{\infty} \frac{b b_{n+k}}{a_{n} \cdots a_{n+k}} & =\frac{b b_{n}}{a_{n}}+\sum_{k=1}^{\infty} \frac{b b_{n+k}}{a_{n+k-1} \cdots a_{n+k}} \cdot \frac{1}{a_{n} \cdots a_{n+k-2}} \\
& <\frac{1}{2}+\frac{1}{4} \sum_{l=0}^{\infty}\left(\frac{1}{2}\right)^{l}=1,
\end{aligned}
$$

a contradiction. Thus $a_{n} \leqq 2 b b_{n}$ for all large $n$.

LEMMA 2.29. If the series (2.28) is rational, say $\xi=a / b$, and $b_{n+1}=o\left(a_{n} a_{n+1}\right)$, then there exists a sequence of positive integers $\left\{c_{n}\right\}$ so that for all large $n$ we have

$$
b b_{n}=c_{n} a_{n}-c_{n+1}, \quad 0<c_{n+1}<a_{n} \text {, and } c_{n+1}=o\left(a_{n}\right) \text {. }
$$

Conversely, if these conditions hold then the series (2.28) is rational. 
Proof. Choose $N$ so that for all $n>N$ we have $b b_{n}<a_{n} a_{n+1} / 4$. Now for $n \geqq N$ choose $c_{n}, c_{n+1}$ so that

$$
\begin{aligned}
& b b_{n}=c_{n} a_{n}-c_{n+1}, \\
& c_{n}>0 \\
& 0<c_{n+1}<a_{n}
\end{aligned}
$$

and $c_{n-1}^{\prime}, c_{n+2}^{\prime}$

$$
b b_{n+1}=c_{n+1}^{\prime} a_{n+1}-c_{n+2}^{\prime}, \quad \begin{array}{r}
c_{n+1}^{\prime}>0 \\
0<c_{n+2}^{\prime}<a_{n+1} .
\end{array}
$$

Then

$$
\begin{aligned}
b a_{1} \cdots a_{n-1} \xi= & a a_{1} \cdots a_{n-1} \\
= & \text { integer }+\frac{b b_{n}}{a_{n}}+\frac{b b_{n+1}}{a_{n} a_{n+1}}+\sum_{k=2}^{\infty} \frac{b b_{n+k}}{a_{n} \cdots a_{n+k}} \\
= & \text { integer }-\frac{c_{n+1}}{a_{n}}+\frac{c_{n+1}^{\prime}}{a_{n}}-\frac{c_{n+2}^{\prime}}{a_{n} a_{n+1}} \\
& +\frac{1}{a_{n}} \sum_{k=2}^{\infty} \frac{b b_{n+k}}{a_{n+1} \cdots a_{n+k}} \\
= & \text { integer }-\frac{c_{n+1}}{a_{n}}+\frac{c_{n+1}^{\prime}}{a_{n}}-\frac{c_{n+2}^{\prime}}{a_{n} a_{n+1}}+\frac{\theta}{a_{n}}, \quad 0<\theta<\frac{1}{2} .
\end{aligned}
$$

Thus

$$
\frac{1}{a_{n}}\left(-c_{n+1}+c_{n+1}^{\prime}-\frac{c_{n+2}^{\prime}}{a_{n+1}}+\theta\right)=\text { integer }
$$

and since $0<c_{n+1}<a_{n}, \quad 0<c_{n+1}^{\prime} \leqq\left[a_{n} / 4\right]+10<c_{n+2}^{\prime} / a_{n+1}<1$, $0<\theta<\frac{1}{2}$, this is possible only if $c_{n+1}=c_{n+1}^{\prime}$.

Now choose $N$ so large that $b b_{n+1}<\varepsilon a_{n} a_{n+1}$ for all $n>N$, then from (2.31) we have

$$
\begin{aligned}
\text { integer } & =-\frac{c_{n+1}}{a_{n}}+\sum_{k=1}^{\infty} \frac{b b_{n+k}}{a_{n} a_{n+1} \cdots a_{n+k}}<-\frac{c_{n+1}}{a_{n}}+\varepsilon \sum_{k=1}^{\infty} \frac{1}{a_{n} \cdots a_{n+k-2}} \\
& \leqq-\frac{c_{n+1}}{a_{n}}+2 \varepsilon .
\end{aligned}
$$

Thus $c_{n+1}<2 \varepsilon a_{n}$ for all $n>N$.

If condition (2.30) holds for all $n \geqq N$ then

$$
\begin{aligned}
\sum_{n=N}^{\infty} \frac{b b_{n}}{a_{1} \cdots a_{n}} & =\sum_{n=N}^{\infty} \frac{c_{n} a_{n}-c_{n+1}}{a_{1} \cdots a_{n}} \\
& =\frac{c_{N}}{a_{1} \cdots a_{N-1}}-\sum_{n=N}^{\infty} c_{n+1}\left(\frac{1}{a_{1} \cdots a_{n}}-\frac{a_{n+1}}{a_{1} \cdots a_{n+1}}\right) \\
& =\frac{c_{N}}{a_{1} \cdots a_{N-1}}
\end{aligned}
$$


is clearly rational.

Finally we need a fact from sieve theory. We are grateful to R. Miech for supplying the correct constants.

LEMma 2.32. Given an integer $a$ and $\varepsilon>0$ then for large $y$ the number of integers $m$ satisfying

$$
m \not \equiv 0, m \not \equiv a(\bmod p)
$$

for all primes $p$, with $2<p<y^{1 / 5}$ exceeds $y^{1-\varepsilon}$.

Proof of Theorem 2.26. Let $f(n)$ stand for either $\sigma(n)$ or $\varphi(n)$ and assume that

$$
\sum_{n=1}^{\infty} \frac{f(n)}{a_{1} \cdots a_{n}}=\frac{a}{b} .
$$

Since $a_{n}>n^{11 / 12}$ for large $n$ the hypothesis of Lemma 2.29 is satisfied and we get

$$
b f(n)=c_{n} a_{n}-c_{n+1} \text { for large } n .
$$

Since $f(n)=o\left(n^{1+\varepsilon}\right)$ for all $\varepsilon>0$ we get

$$
c_{n}<n^{1 / 12+\varepsilon} \text { for large } n \text {. }
$$

From Lemma 2.28 we get

$$
a_{n}=O(f(n))=O\left(n^{1+\varepsilon}\right)
$$

and hence the number of integers $n \leqq x$ for which

$$
\frac{a_{n+1}}{a_{n}}>1+x^{-1 / 2}
$$

is $O\left(x^{3 / 4}\right)$, since otherwise we would have

$$
a_{x}=\prod_{n<x} \frac{a_{n+1}}{a_{n}}>\left(1+x^{-1 / 2}\right)^{x^{3 / 4}}>x^{2}
$$

for large $x$, in contradiction to (2.35). From now on we restrict our attention to integers $n$ for which

$$
\frac{a_{n+1}}{a_{n}}<1+n^{-1 / 2} .
$$

For such integers we get from (2.33) and (2.35) that 


$$
\begin{aligned}
\frac{f(n+1)}{f(n)} & =\frac{c_{n+1} a_{n+1}}{c_{n} a_{n}}\left(1-\frac{c_{n+2}}{c_{n+1} a_{n+1}}\right) /\left(1-\frac{c_{n+1}}{c_{n} a_{n}}\right) \\
& =\frac{c_{n+1}}{c_{n}}\left(1+O\left(n^{-1 / 2}\right)\right)\left(1+O\left(n^{-3 / 4+\varepsilon}\right)\right) \\
& =\frac{c_{n+1}}{c_{n}}+O\left(n^{-1 / 2+\varepsilon}\right)
\end{aligned}
$$

Now consider a prime $q, \frac{1}{2} x^{1 / 11} \leqq q \leqq x^{1 / 11}$, then according to Lemma 2.32 there exist more than $y^{1-\varepsilon}$ integers $m \leqq y=x^{10 / 11}$ so that

$$
m \not \equiv 0, m \not \equiv-2 q(\bmod p)
$$

for all primes $p$ with $2<p<y^{1 / 5}$. We may even assume that $m$ is odd. The number of integers $n=2 q m$ where $m$ satisfies (2.38) exceeds $x^{10 / 11-\varepsilon}>x^{3 / 4}$ and hence we can pick such an $n$ that satisfies (2.37) with $x / 2 \leqq n \leqq x$.

Now

$$
f(n)=f(2 q) f(m)
$$

where

$$
\frac{f(2 q)}{2 q}= \begin{cases}\frac{3(q+1)}{2 q} & \text { if } f=\sigma \\ \frac{q-1}{2 q} & \text { if } f=\varnothing\end{cases}
$$

in either case

$$
f(2 q)=A / q, \quad A \text { an integer not divisible by } q .
$$

Since $m$ has at most 5 prime factors all exceeding $y^{1 / 5}$ we have

$$
\left(1-y^{-1 / 5}\right)^{5}<\frac{f(m)}{m}<\left(1+y^{-1 / 5}\right)^{5}
$$

$$
f(m)=m\left(1+O\left(y^{-1 / 5}\right)\right)=m\left(1+O\left(x^{-2 / 11}\right)\right) .
$$

By the same reasoning we get

$$
f(n+1)=n\left(1+O\left(x^{-2 / 11}\right)\right) .
$$

Substituting (2.39), (2.40) and (2.41) in (2.37) we get

$$
\frac{f(n+1)}{f(n)}=\frac{A}{q}\left(1+O\left(x^{-2 / 11}\right)\right)=\frac{c_{n+1}}{c_{n}}+O\left(x^{-1 / 2+\varepsilon}\right) .
$$

But since $q>x^{1 / 12}$ and $c_{n}<x^{1 / 12}$ we get

$$
\frac{1}{q c_{n}} \leqq\left|\frac{A}{q}-\frac{c_{n+1}}{c_{n}}\right|<x^{-2 / 11+\varepsilon} .
$$


Since $q c_{n}<x^{1 / 11+1 / 12}<x^{2 / 11-\varepsilon}$ this leads to a contradiction.

We could get similar irrationality results if the functions $\sigma(n)$ or $\varphi(n)$ are replaced by $\sigma_{k}(n)(k \geqq 1)$ or products of powers of $\sigma_{k}(n)$ and $\varphi(n)$. In each case we would need the assumption that the $a_{n}$ are monotonic, increasing faster than a certain fractional power of the numerators.

From Lemma 2.29 it is clear that there is a set of power $2^{\mathrm{No}}$ of series (2.25) which are rational even if we restrict the integers $c_{n}$ to the values 1 or 2 since for $c_{n}=1$ we can choose $a_{n}=\sigma(n)-1$ or $\sigma(n)-2$ to get $c_{n+1}=1$ or 2 respectively and for $c_{n}=2$ we choose $a_{n}=[(\sigma(n)-1) / 2]$ to get $c_{n+1}=1$ if $\sigma(n)$ is odd and $c_{n+1}=2$ if $\sigma(n)$ is even. For the series with numerators $\varphi(n)$ we would have to use $c_{n}=1,2$ or 3 since all $\varphi(n)$ are even for $n>2$.

\section{REFERENCES}

1. P. Erdös, On arithmetical properties of Lambert series, J. Indian Math. Soc., 12 (1948), 63-66.

2. P. Erdös and H. Heilbronn, On the addition of residue classes mod $p$, Acta Arithmetica 9 (1964), 149-159.

3. M. Kac, Note on the distribution of values of the arithmetic function $d(m)$, Bull. Amer. Math. Soc., 47 (1941), 815-817.

4. E. G. Straus, On a problem in conbinatorial number theory, J. Math. Sci., (Delhi), 1 (1966), 77-80.

$5 . \quad$ Some problems concerning sum-free and average-free sets, Lecture notes, Summer institute on Number Theory, Stony Brook, N.Y. 1969, Cll.

Received may 27, 1970. This work was supported under grant No. GP-13164

University of CAlifornia, Los ANgeles 


\title{
PACIFIC JOURNAL OF MATHEMATICS
}

\section{EDITORS}

H. SAMELSON

Stanford University

Stanford, California 94305

C. R. HobвY

University of Washington

Seattle, Washington 98105

\section{J. DugunduI}

Department of Mathematics

University of Southern California

Los Angeles, California 90007

RICHARD ARENS

University of California

Los Angeles, California 90024

\section{ASSOCIATE EDITORS}
E. F. BECKENBACH
B. H. Neumann
F. WOLE
K. Yoshida

\section{SUPPORTING INSTITUTIONS}

\author{
UNIVERSITY OF BRITISH COLUMBIA \\ CALIFORNIA INSTITUTE OF TECHNOLOGY \\ UNIVERSITY OF CALIFORNIA \\ MONTANA STATE UNIVERSITY \\ UNIVERSITY OF NEVADA \\ NEW MEXICO STATE UNIVERSITY \\ OREGON STATE UNIVERSITY \\ UNIVERSITY OF OREGON \\ OSAKA UNIVERSITY \\ UNIVERSITY OF SOUTHERN CALIFORNIA
}

\author{
STANFORD UNIVERSITY \\ UNIVERSITY OF TOKYO \\ UNIVERSITY OF UTAH \\ WASHINGTON STATE UNIVERSITY \\ UNIVERSITY OF WASHINGTON

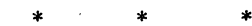 \\ AMERICAN MATHEMATICAL SOCIETY \\ CHEVRON RESEARCH CORPORATION \\ NAVAL WEAPONS CENTER
}

The Supporting Institutions listed above contribute to the cost of publication of this Journal, but they are not owners or publishers and have no responsibility for its content or policies.

Mathematical papers intended for publication in the Pacific Journal of Mathematics should be in typed form or offset-reproduced, (not dittoed), double spaced with large margins. Underline Greek letters in red, German in green, and script in blue. The first paragraph or two must be capable of being used separately as a synopsis of the entire paper. The editorial "we" must not be used in the synopsis, and items of the bibliography should not be cited there unless absolutely necessary, in which case they must be identified by author and Journal, rather than by item number. Manuscripts, in duplicate if possible, may be sent to any one of the four editors. Please classify according to the scheme of Math. Rev. Index to Vol. 39. All other communications to the editors should be addressed to the managing editor, Richard Arens, University of California, Los Angeles, California, 90024.

50 reprints are provided free for each article; additional copies may be obtained at cost in multiples of 50 .

The Pacific Journal of Mathematics is published monthly. Effective with Volume 16 the price per volume (3 numbers) is $\$ 8.00$; single issues, $\$ 3.00$. Special price for current issues to individual faculty members of supporting institutions and to individual members of the American Mathematical Society: $\$ 4.00$ per volume; single issues $\$ 1.50$. Back numbers are available.

Subscriptions, orders for back numbers, and changes of address should be sent to Pacific Journal of Mathematics, 103 Highland Boulevard, Berkeley, California, 94708.

PUBLISHED BY PACIFIC JOURNAL OF MATHEMATICS, A NON-PROFIT CORPORATION

Printed at Kokusai Bunken Insatsusha (International Academic Printing Co., Ltd.), 7-17, Fujimi 2-chome, Chiyoda-ku, Tokyo, Japan. 


\section{Pacific Journal of Mathematics}

\section{Vol. 36, No. $3 \quad$ BadMonth, 1971}

E. M. Alfsen and B. Hirsberg, On dominated extensions in linear subspaces of

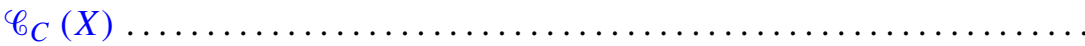

Joby Milo Anthony, Topologies for quotient fields of commutative integral

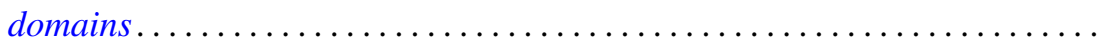

V. Balakrishnan, G. Sankaranarayanan and C. Suyambulingom, Ordered cycle lengths in a random permutation .......................... 603

Victor Allen Belfi, Nontangential homotopy equivalences............... 615

Jane Maxwell Day, Compact semigroups with square roots .............. 623

Norman Henry Eggert, Jr., Quasi regular groups of finite commutative nilpotent algebras .......................................... 631

Paul Erdős and Ernst Gabor Straus, Some number theoretic results ......... 635

George Rudolph Gordh, Jr., Monotone decompositions of irreducible Hausdorff continua .............................................

Darald Joe Hartfiel, The matrix equation $A X B=X \ldots \ldots \ldots \ldots \ldots \ldots . \ldots 69$

James Howard Hedlund, Expansive automorphisms of Banach spaces. II . . . . 671

I. Martin (Irving) Isaacs, The p-parts of character degrees in p-solvable

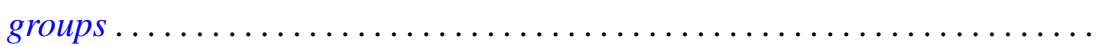

Donald Glen Johnson, Rings of quotients of $\Phi$-algebras ............... 693

Norman Lloyd Johnson, Transition planes constructed from semifield planes....

Anne Bramble Searle Koehler, Quasi-projective and quasi-injective modules.

James J. Kuzmanovich, Completions of Dedekind prime rings as second endomorphism rings...

B. T. Y. Kwee, On generalized translated quasi-Cesàro summability ...

Yves A. Lequain, Differential simplicity and complete integral closure

741

Mordechai Lewin, On nonnegative matrices.

753

Kevin Mor McCrimmon, Speciality of quadratic Jordan algebras ...

Hussain Sayid Nur, Singular perturbations of differential equations in abstract spaces .....................................

D. K. Oates, A non-compact Krein-Milman theorem .

Lavon Barry Page, Operators that commute with a unilateral shift on an invariant subspace...

Helga Schirmer, Properties of fixed point sets on dendrites.

Saharon Shelah, On the number of non-almost isomorphic models of $T$ in a

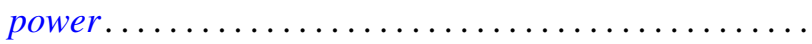

Robert Moffatt Stephenson Jr., Minimal first countable Hausdorff spaces....

Masamichi Takesaki, The quotient algebra of a finite von Neumann algebra 